\title{
Interaction with adipocytes induces lung adenocarcinoma A549 cell migration and tumor growth
}

\author{
FAN-FAN LI ${ }^{*}$, HANG ZHANG ${ }^{*}$, JING-JING LI, YA-NAN CAO, XIANG DONG and CONG GAO \\ Department of Oncology, The Second Affiliated Hospital of Anhui Medical University, Hefei, Anhui 230601, P.R. China
}

Received November 22, 2017; Accepted April 23, 2018

DOI: $10.3892 / \mathrm{mmr} .2018 .9226$

\begin{abstract}
Adipocytes have been demonstrated to promote the progression of various tumors through modulation of cancer cell metabolism. However, their role in lung cancer progression remains undetermined. In the present study, adipocytes and lung adenocarcinoma A549 cells were cultured in a Transwell co-culture system. Cancer cells were additionally cultured in conditioned medium, obtained from adipocytes or co-cultured cells. A MTT and colony formation assay were performed to assess A549 cell proliferation. The expression of epithelial-mesenchymal transition protein markers E-cadherin and vimentin were measured by western blotting. A549 cell migration and invasion was determined with wound healing, Transwell and Matrigel assays. Oil Red-O staining was used to evaluate intracellular lipid content. Colorimetric assays were utilized to detect free fatty acid, glucose uptake, lactate production and triglyceride content in cells. The results revealed a reciprocal interaction between adipocytes and A549 cells, which significantly enhanced A549 cell proliferation and metastasis; whereas, the expression of E-cadherin was decreased and vimentin was increased in A549 cells. Additionally, A549 cells exhibited metabolic reprogramming in vitro following co-culture with adipocytes. It was demonstrated that lipid droplets accumulation, glucose consumption and lactate production increased in tumor cells exposed to adipocytes. Furthermore, adipocytes co-cultured with A549 cells exhibited a decrease in the number and size of lipid droplets, a decrease in the intracellular triglyceride content and a significant increase in the release of free fatty acids. These findings highlighted the crucial role of adipocytes in the modulation of lung adenocarcinoma A549 cell metabolism and suggested the involvement of adipocytes in lung cancer progression.
\end{abstract}

Correspondence to: Professor Fan-Fan Li, Department of Oncology, The Second Affiliated Hospital of Anhui Medical University, 678 Furong Road, Hefei, Anhui 230601, P.R. China E-mail: fflahykdx@126.com

${ }^{*}$ Contributed equally

Key words: lung adenocarcinoma, adipocytes, proliferation, migration, metabolism

\section{Introduction}

At present, lung cancer is the most common cause of cancer-associated mortality worldwide (1). Lung adenocarcinoma is most frequently diagnosed, which has a high 5-year relative mortality rate in the range $51-99 \%$, due to the presence of metastasis at the time of diagnosis (2). Studies of relative risk factors and a deeper understanding of adenocarcinoma pathogenesis are required to improve prevention strategies and targeted treatments.

Recent epidemiological and experimental studies have identified adipose tissue and associated metabolic abnormalities as negative prognostic predictors $(3,4)$. Adipocytes represent the primary percentage of cells within adipose tissue (5). Previous studies suggested that an interaction between adipocytes and certain cancer types, including melanoma, prostate, ovarian and breast cancer, is critical for tumor cell growth and invasion (6-9). Adipocytes are a source of adipokines, including leptin, adiponectin and visfatin, in addition to growth, immune, inflammatory and angiogenic factors. Numerous studies have demonstrated that adipocytes are involved in the regulation of systemic energy and metabolic homeostasis $(6-8,10,11)$. In addition, research has demonstrated that in the presence of cancer cells, which may act as metabolic parasites, adipocytes become highly metabolically active and secrete significant quantities of metabolic substrates, including glycerol and fatty acids. These metabolites are utilized as the macromolecular building blocks to support cancer cell proliferation and provide the energy required for biomass synthesis, migration and invasion (9).

The Warburg effect describes the high levels of aerobic glycolysis observed in cancer cells. This provides their primary energy source, by incompletely utilizing glucose and switching to upstream intermediates for biosynthesis, providing cancer cells with materials for the synthesis of essential cellular components, including macromolecules $(12,13)$. In addition, the Warburg effect is thought to accelerate lactate secretion, thereby acidifying the surrounding extracellular matrix and facilitating angiogenesis and tumor metastasis (14). Furthermore, previous studies have suggested that lipids may enhance the Warburg effect in certain cancer cells $(15,16)$.

The interaction between cancer cells and adipocytes in lung cancer progression remains unclear. Although lung cancer is not considered to be associated with obesity, previous studies have demonstrated that obesity induced by a high-fat diet increases cancer-associated mortality and the likelihood 
of lung carcinoma in mice $(17,18)$. Bone, in which adipocytes may increase due to aging or metabolic disorder, is a common site of metastasis in lung cancer (19). Other common sites of metastasis in lung cancer include the liver, the central organ of lipid metabolism and catabolism (20), and the brain, $60 \%$ of which is composed of lipids $(21,22)$. The role of adipocytes and their metabolic substrates should be considered in the progression of lung cancer metastasis, according to the seed and soil theory, which was first proposed by Stephen Paget, and suggests that metastatic homing of tumor cells is not a stochastic event; however, is governed by the interaction between metastatically competent cancer cells (the 'seed') and the permissive microenvironment of specific organs (the 'soil') (23). In the present study, the potential capacity of adipocytes to promote the growth and metastasis of lung adenocarcinoma A549 cells was determined and the mechanisms responsible for the observed effects were investigated. The present study of the association between adipocytes and lung cancer cells may provide insight to the underlying progression mechanism, and a novel target to cure lung cancer.

\section{Materials and methods}

Cell lines and reagents. 3T3-L1 pre-adipose and human lung adenocarcinoma A549 cells were obtained from Fudan University (Shanghai, China). Dulbecco's modified Eagle's medium (DMEM) was purchased from Hyclone (GE Healthcare Life Sciences, Logan, UT, USA). Fetal bovine serum (FBS) and newborn calf serum (NBCS) were purchased from Zhejiang Tianhang Biotechnology Co., Ltd. (Huzhou, China); penicillin/streptomycin was purchased from Gibco (Thermo Fisher Scientific, Inc., Waltham, MA, USA). Dexamethasone, 3-isobutyl-1-methylxanthine (IBMX) and dimethyl sulfoxide (DMSO) were purchased from Sigma-Aldrich (Merck KGaA, Darmstadt, Germany). Insulin was purchased from Beijing Solarbio Science \& Technology Co., Ltd. (Shanghai, China). Crystal violet, MTT and bovine serum albumin (BSA) were purchased from Amresco, LLC (Solon, OH, USA). The reagents associated with western blot analysis were purchased from Beyotime Institute of Biotechnology (Haimen, China). Polyvinylidene difluoride (PVDF) membranes were purchased from EMD Millipore (Billerica, MA, USA).

Cell cultures and adipocyte differentiation. A549 cells were maintained in DMEM supplemented with $10 \%$ FBS and $1 \%$ penicillin/streptomycin. 3T3-L1 cells were cultured in DMEM supplemented with $10 \%$ NBCS and $1 \%$ penicillin/streptomycin. Cultures were maintained in a humidified atmosphere of $95 \%$ air and $5 \% \mathrm{CO}_{2}$ at $37^{\circ} \mathrm{C}$. 3T3-L1 pre-adipocytes were harvested and allowed to reach $100 \%$ confluence. Following 2 days, cell differentiation was induced by the addition of a hormonal mixture composed of $10 \mu \mathrm{g} / \mathrm{ml}$ insulin, $1 \mu \mathrm{M}$ dexamethasone and $0.5 \mathrm{mM}$ IBMX in DMEM with $10 \%$ FBS. Following a further 2 days, the induction medium was replaced by DMEM supplemented with $10 \%$ FBS and $10 \mu \mathrm{g} / \mathrm{ml}$ insulin only. The medium was subsequently replaced at 2-day intervals.

Production of conditioned medium (CM). CM was collected from adipocytes cultured alone at $37^{\circ} \mathrm{C}$ with serum-free
DMEM containing 1\% BSA for $24 \mathrm{~h}$ (Ad-CM), or obtained from adipocytes previously co-cultured during $48 \mathrm{~h}$ with A549 cells in complete medium, cells were subsequently incubated in serum-free DMEM containing $1 \% \mathrm{BSA}$ at $37^{\circ} \mathrm{C}$ for $24 \mathrm{~h}$ (Ad-CCM). The CM was subsequently harvested and centrifuged at $550 \mathrm{xg}$ at room temperature (RT) for $5 \mathrm{~min}$ to remove cellular debris, and stored at $-80^{\circ} \mathrm{C}$ until use.

Oil Red-O staining. The differentiated adipocytes, A549 cells, co-cultured adipocytes and co-cultured A549 cells were washed twice with $500 \mu 1$ PBS and subsequently fixed with $400 \mu 14 \%$ paraformaldehyde at RT for $30 \mathrm{~min}$. Cells were subsequently removed and washed twice with PBS, prior to the addition of $400 \mu \mathrm{l}$ Oil Red-O staining solution to each well for $30 \mathrm{~min}$ at RT. Following washing with $60 \%$ isopropyl alcohol, cells were observed under a light microscope (Olympus Corporation, Tokyo, Japan; magnification, x100 and x400).

Indirect co-culture. Adipocytes were indirectly co-cultured with A549 cells using a Transwell culture system (pore size, $0.4 \mu \mathrm{m}$; Corning Incorporated, Corning, NY, USA), which were maintained in DMEM with $10 \% \mathrm{FBS}$ at $37^{\circ} \mathrm{C}$. To assess adipocyte biology, the pre-adipocytes were seeded at $1 \times 10^{5} / \mathrm{ml}$ in the bottom, and maintained until the confluency reached $100 \%$ and differentiation occurred. The adipocytes were obtained 8 days following differentiation. The intact adipocytes were co-cultured with the A549 cells, which were seeded in the upper chamber at $1 \times 10^{5}$ cells $/ \mathrm{ml}$. The time of co-culture was 0,3,6 and 9 days, respectively. Conversely, for experiments assessing cancer cell biology, adipocytes were grown in the upper chamber, with A549 cells in the bottom. Adipocytes or cancer cells cultured alone served as controls.

Cell proliferation assay. The proliferative ability of A549 cells was measured by an MTT assay. A549 cells were seeded in 96-well plates at $2 \times 10^{3}$ cells/well, allowed to adhere $(0 \mathrm{~h})$ and subsequently cultured in the presence of Ad-CM, Ad-CCM, or DMEM with $10 \%$ FBS at $37^{\circ} \mathrm{C}$ for 24,48 or $72 \mathrm{~h}$. MTT (20 $\mu \mathrm{l}$; $5 \mathrm{mg} / \mathrm{ml}$ ) was added to each well and incubated at $37^{\circ} \mathrm{C}$ for $4 \mathrm{~h}$. The medium was aspirated and cells were treated with $150 \mu \mathrm{l}$ DMSO at RT for $10 \mathrm{~min}$. Following this, absorbance was measured with a microplate reader (SpectraMax 190; Molecular Devices, LLC, Sunnyvale, CA, USA) at $490 \mathrm{~nm}$.

Colony formation assay. For colony formation assays, A549 cells were plated into 6 -well plates $\left(1 \times 10^{3}\right.$ cells/well $)$, maintained in DMEM with 10\% FBS and allowed to adhere for $24 \mathrm{~h}$. Culture medium was subsequently altered to $10 \%$ FBS in DMEM, Ad-CM or Ad-CCM and the plate was incubated in a $5 \% \mathrm{CO}_{2}$ incubator at $37^{\circ} \mathrm{C}$. Subsequently, the culture medium was replaced every 4 days at $50 \%$. Following 18 days, cells were washed twice with PBS and fixed with $4 \%$ paraformaldehyde at RT for $20 \mathrm{~min}$. Colonies were stained with $0.1 \%$ crystal violet at RT for $15 \mathrm{~min}$. Colonies were counted under a light microscope (Olympus Corporation; magnification, x100).

Western blot analysis. Following treatment, the A549 cells were harvested and lysed using lysis buffer (Beyotime Institute of Biotechnology) for 30 min on ice. The total proteins were isolated by centrifuging at $12,000 \mathrm{x} \mathrm{g}$ for $10 \mathrm{~min}$ at $4^{\circ} \mathrm{C}$ 
and quantified using a bicinchoninic acid protein assay kit (Beyotime Institute of Biotechnology). A total of $30 \mu \mathrm{g}$ total protein was separated on $10 \%$ SDS-PAGE and transferred to PVDF membranes. The membranes were subsequently blocked in 5\% non-fat milk at RT for $2 \mathrm{~h}$. Following washing with Tris-buffered saline with $0.05 \%$ Tween-20 (TBST), the membranes were incubated with the corresponding primary antibodies, including Rabbit anti-E-cadherin $(1: 10,000$; cat. no. ab40772), anti-Vimentin (1:1,000; cat. no. ab92547) and anti- $\beta$-catenin (1:5,000; cat. no. ab32572; all Abcam, Cambridge, UK) at $4{ }^{\circ} \mathrm{C}$ overnight. Subsequently, the membranes were probed with the appropriate horseradish peroxidase (HRP)-conjugated secondary antibodies, goat anti-rabbit immunoglobulin $\mathrm{G}$ heavy and light HRP $(1: 2,500$; cat. no. ZB2301; OriGene Technologies, Inc., Rockville, $\mathrm{MD}$, USA) for $2 \mathrm{~h}$ at room temperature. Following washing in TBST, the immunoreactive proteins were visualized using an enhanced chemiluminescent kit (Pierce; Thermo Fisher Scientific, Inc.).

Wound closure assay. A wound closure assay was conducted to assess the migration of A549 cells. When cell confluence reached $80 \%$, a wound was made in the center of the cell monolayer using a $10 \mu \mathrm{l}$ sterile pipette tip. Following co-culture, cell migration was observed using a light microscope (Olympus Corporation; magnification, x100). Images were captured at 0 and $24 \mathrm{~h}$ following when the wound was made and the ImageJ version $1.44 p$ software (National Institutes of Health, Bethesda, MD, USA) was used to quantify the wound width $(\mu \mathrm{m})$. The wound-healing rate was calculated as follows: (width at $0 \mathrm{~h}$-width following $24 \mathrm{~h}$ )/width at $0 \mathrm{~h}$ x100.

Transwell migration and invasion assay. To detect cell migration, Transwell chambers (Corning Incorporated) with a polycarbonate membrane ( 24 wells; pore size, $8 \mu \mathrm{m}$ ) were used. A549 cells were cultured in DMEM, Ad-CM or Ad-CCM with $10 \% \mathrm{FBS}$ for $48 \mathrm{~h}$ at $37^{\circ} \mathrm{C}$, trypsinized and resuspended in serum-free DMEM at a density of $1 \times 10^{5}$ cells $/ \mathrm{ml}$. Cell suspension $(200 \mu \mathrm{l})$ was added to the upper chamber and DMEM supplemented with $10 \%$ FBS $(600 \mu \mathrm{l})$ was added to the lower chamber as a chemoattractant. A549 cells cultured alone in similar conditions served as a control. To detect cell invasion, filters were coated with $50 \mu \mathrm{g} / \mathrm{ml}$ Matrigel (BD Biosciences, Franklin Lakes, NJ, USA) solution. Matrigel was not used for cell migration assays. Following $24 \mathrm{~h}$, cells were washed twice with PBS, fixed in $4 \%$ paraformaldehyde at RT for $30 \mathrm{~min}$, and stained with $0.1 \%$ crystal violet for $30 \mathrm{~min}$ at RT. The number of migrated or invaded cells were counted under a microscope (Olympus Corporation; magnification, x200). Means were obtained from five randomly selected fields in each well.

Free fatty acid and triglyceride (TG) detection. To detect the free fatty acid levels in each media, CM was obtained from adipocytes cultured alone or indirectly co-cultured with A549 cells at $37^{\circ} \mathrm{C}$ and subsequently analyzed using a non-esterified fatty acid quantification kit (cat. no. A042-1; Nanjing Jiancheng Bioengineering Institute, Nanjing, China) under a microplate reader (SpectraMax 190; Molecular Devices, LLC) at $440 \mathrm{~nm}$, according to the manufacturer's protocol. To detect intracellular TGs, following indirect co-culture, adipocytes or A549 cells were centrifuged at $550 \mathrm{x} \mathrm{g}$ for $10 \mathrm{~min}$ at RT, and sonicated at $300 \mathrm{~W}, 5 \mathrm{sec} /$ beat, $30 \mathrm{sec}$ of interval, 3-5 repeats in an ice bath. The intracellular TG content was determined using a glycerol-3-phosphate oxidase/phenol + aminophenazone enzymatic kit (cat. no. A110-1; Nanjing Jiancheng Bioengineering Institute), according to the manufacturer's protocol. The TG and FFA content were determined at 510 and $440 \mathrm{~nm}$, respectively, under a microplate reader (SpectraMax 190; Molecular Devices, LLC).

Detection of glucose consumption and lactate production. For the detection of glucose and lactate concentrations, $1 \times 10^{5}$ A549 cells $/ \mathrm{ml}$ were seeded in 6-well plates and cultured in DMEM with $10 \% \mathrm{FBS}$ at $37^{\circ} \mathrm{C}$. When cells had reached $60-70 \%$ confluence, the culture medium was subsequently altered to $10 \%$ FBS in DMEM, Ad-CM or Ad-CCM and the plate was incubated in a $5 \% \mathrm{CO}_{2}$ incubator at $37^{\circ} \mathrm{C}$. Following $24 \mathrm{~h}$, the culture supernatant was collected for the detection of glucose and lactate. Glucose (cat. no. F006) and lactate (cat. no. A019-2) concentrations were determined using corresponding kits (Nanjing Jiancheng Bioengineering Institute) and a microplate reader (SpectraMax 190; Molecular Devices, LLC) at $505 \mathrm{~nm}$ and $530 \mathrm{~nm}$, respectively, according to the respective manufacturer's protocol. The number of cells in each well was simultaneously counted using the blood cell counting chamber. Glucose consumption and lactate production were normalized to cell number.

Statistical analysis. All experimental data are presented as the mean \pm standard error of the mean from at least three independent experiments. Statistical analysis of the data was performed using GraphPad Prism 7.0 (GraphPad Software, Inc., La Jolla, CA, USA). Student's t-test was used for comparisons between two groups, while multiple groups were compared using one-way analysis of variance followed by Tukey's post-hoc test. $\mathrm{P}<0.05$ was considered to indicate a statistically significant difference.

\section{Results}

Effect of adipocytes on the proliferation and colony formation of lung adenocarcinoma A549 cells. In the present study, a 3T3-L1 pre-adipose cell line was used to generate mature adipocytes, according to a standard differentiation protocol (24). Following induction and differentiation, a large number of lipid droplets had accumulated and were clearly visible in the cytoplasm of adipocytes when stained with Oil Red-O (Fig. 1A). To assess the potential role of adipocytes in A549 cell growth, MTT and colony formation assays were conducted to examine alterations in A549 cell proliferation. A549 cells were grown in the presence of $\mathrm{CM}$ obtained from adipocytes cultured alone (Ad-CM; Fig. 1B), or CM obtained from adipocytes previously co-cultured with A549 cells (Ad-CCM; Fig. 1C). The proliferation rate of A549 cells cultured in Ad-CM was not significantly different from that of A549 cells grown in normal medium (Fig. 1D and E). Notably, as presented in Fig. 1D, Ad-CCM induced a time-dependent increase in A549 cell proliferation. Proliferation was significantly increased compared with cancer cells induced by control medium $(\mathrm{P}<0.01)$ and Ad-CM $(\mathrm{P}<0.05)$. In addition, there was a 1.47 -fold and 1.38 -fold increase in 
A

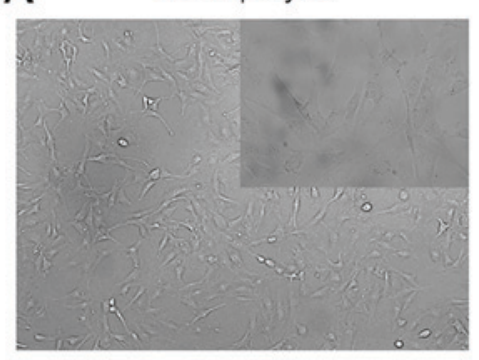

B

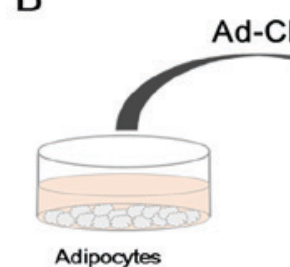

E

A549 cells
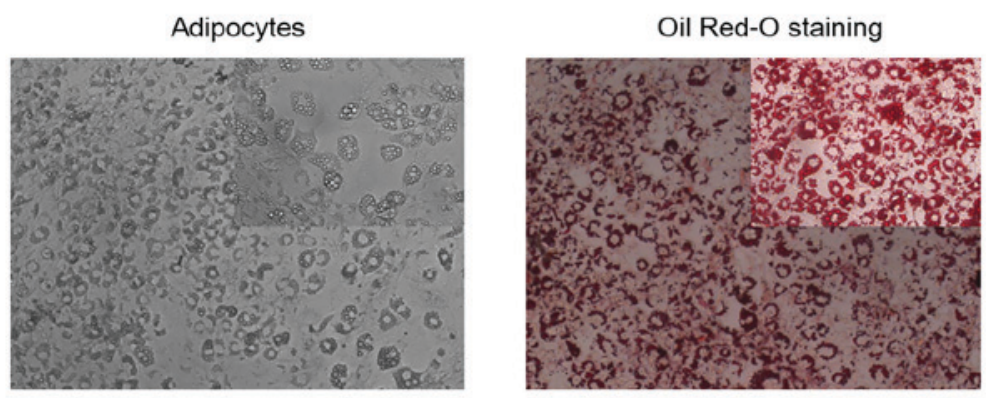

C

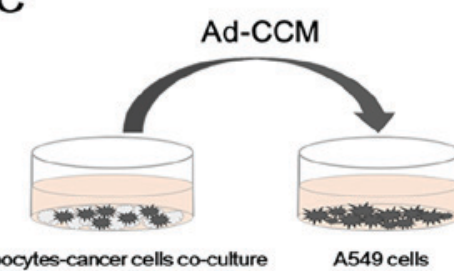

Adipocytes-cancer cells co-culture

A549 cells
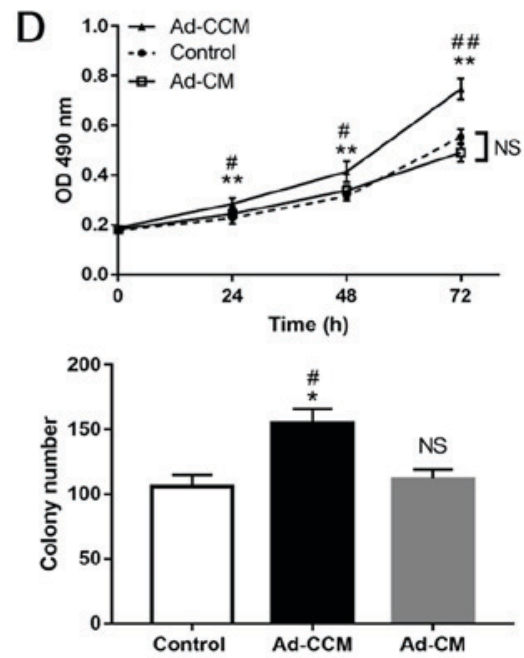

Control

Ad-CCM

Ad-CM

Figure 1. Differentiation of mature adipocytes and the role of adipocyte differentiation in lung adenocarcinoma A549 cell growth. (A) Adipocyte differentiation of 3T3-L1 pre-adipocytes and Oil Red-O staining of mature adipocytes following induction and differentiation. Magnification, x100. Smaller images in the upper right corner are enlarged photos of cells and reveal large lipid droplets in adipocytes. Magnification, x400. (B) Schematic representation of cancer cells treated with CM from adipocytes and (C) the adipocyte-cancer cell co-culture system. (D) An MTT assay was conducted to detect A549 cell proliferation. (E) A colony formation assay was conducted to detect the colony formation ability of A549 cells. ${ }^{*} \mathrm{P}<0.05$, $^{* *} \mathrm{P}<0.01$ vs. respective control; ${ }^{\#} \mathrm{P}<0.05$, "\# $\mathrm{P}<0.01$ vs. Ad-CM. CM, conditioned medium; Ad-CM, adipocyte conditioned medium; Ad-CCM, co-cultured adipocyte conditioned medium; NS, not significant; OD, optical density.

colony formation in lung adenocarcinoma A549 cells cultured in Ad-CCM, compared with cancer cells cultured in control medium and Ad-CM, respectively (Fig. 1E; $\mathrm{P}<0.05$ ). These results suggested that crosstalk between these two cell populations may serve an important role in the proliferation of lung adenocarcinoma A549 cells.

Effects of adipocytes on the EMT, migration and invasion of lung adenocarcinoma A549 cells. To assess the influence of adipocytes on cancer cell metastasis, A549 cells were incubated with $\mathrm{CM}$ as described above. The results indicated that following induction with Ad-CCM for $48 \mathrm{~h}$, the expression level of epithelial cell protein marker E-cadherin was decreased and the mesenchymal protein marker vimentin was increased in A549 cells (Fig. 2A). Furthermore, compared with the control group, a 1 .7-fold increase in the wound-closure rate of A549 cells was observed in the presence of Ad-CCM (Fig. 2B and C; $\mathrm{P}<0.05)$. Similarly, Ad-CCM promoted cancer cell migration studied as compared with the control, as evidenced by the increased numbers of migrated cells in the Transwell migration assay (Fig. 2D and E; P<0.001). In the invasion assay, a 1.86-fold increase in the number of invaded A549 cells was observed in the Ad-CCM cultured with cancer cells, compared with cells grown in control medium (Fig. 2F and G; P<0.001). Nevertheless, A549 cell metastasis was not significantly increased when grown in the presence of Ad-CM (Fig. 2). Collectively, these observations clearly demonstrated that the metastatic phenotype of lung adenocarcinoma A549 cells was enhanced following a reciprocal interaction between adipose and cancer cells.

Co-cultured adipocytes exhibit a lipolysis phenotype in vitro. Cancer cell proliferation and metastasis are energy-intensive processes (25), and the results of the present study indicated that Ad-CCM conferred a proliferative advantage on lung adenocarcinoma A549 cells and enhanced its invasive phenotype. As adipocytes store energy in the form of triglycerides (25), it was hypothesized that this may be the result of energy-dense lipid or metabolic substrate transfer from adipocytes to A549 cells to promote migration and invasion. Therefore, to determine the effect of lung cancer cells on adipocytes, adipocyte metabolism was assessed in a Transwell culture system. As presented in Fig. 3A, mature adipocytes were indirectly co-cultured with lung cancer cells for a prolonged period. As presented in Fig. 3A, the adipocytes exhibited a marked reduction in the number and size of lipid droplets. Additionally, a significant reduction in adipocyte TG content was observed following co-culture with lung cancer cells (Fig. 3B; $\mathrm{P}<0.05$ ). In addition, in the presence of lung cancer cells, adipocytes released significantly more free fatty acids, compared with adipocytes cultured alone (Fig. 3C; $\mathrm{P}<0.001$ ). Taken together, 


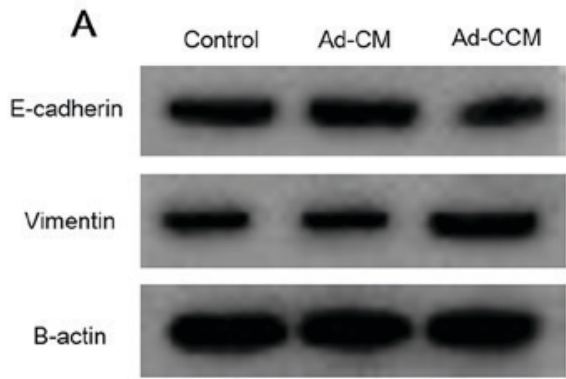

B

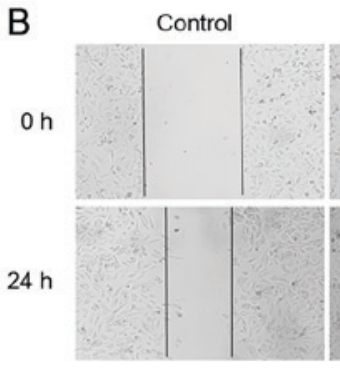

D

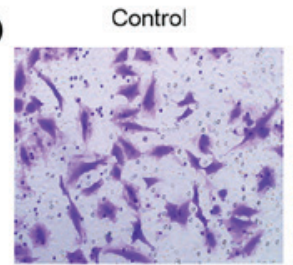

$\mathrm{F}$

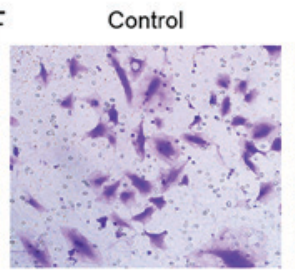

Ad-CM

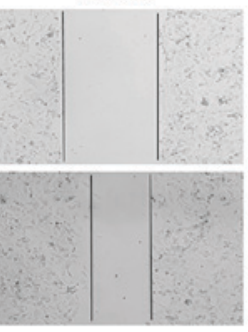

Ad-CM

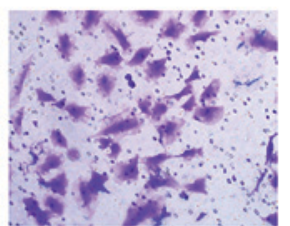

Ad-CM

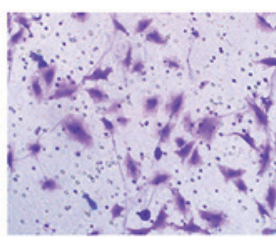

Ad-CCM

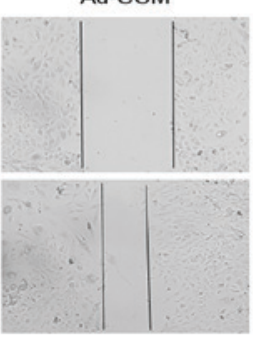

Ad-CCM

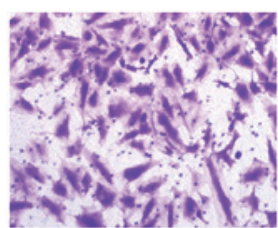

Ad-CCM

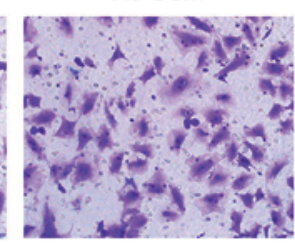

C

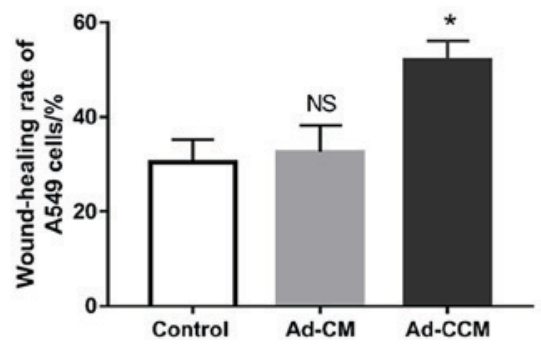

$E$

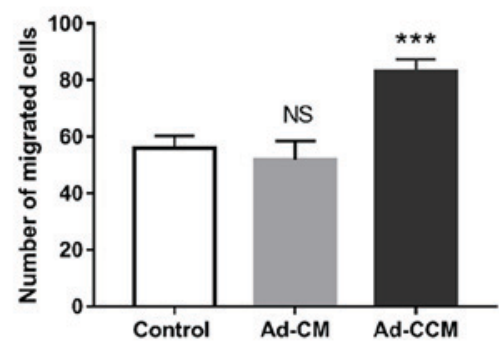

G

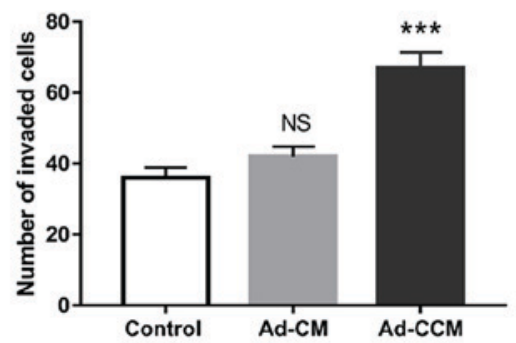

Figure 2. Effect of adipocytes on the migration and invasion of lung adenocarcinoma A549 cells. A549 cells were cultured in normal medium, Ad-CM or Ad-CCM. (A) The expression levels of E-cadherin and vimentin were examined by western blotting. (B) Representative images of the wound closure assay. Magnification, x100. (C) Wound healing rate was calculated. (D) Transwell migration assays were conducted and (E) the migrated cell number was determined. (F) The invasive capacity of A549 cells was investigated in a Transwell invasion assay and (G) invaded cell number was determined. Magnification, x200. ${ }^{*} \mathrm{P}<0.05,{ }^{* * *} \mathrm{P}<0.001$ vs. control. Ad-CM, adipocyte conditioned medium; Ad-CCM, co-cultured adipocyte conditioned medium; NS, not significant.

these findings suggested that A549 cells may have induced adipocyte lipolysis.

Co-cultured lung cancer cells exhibit metabolic reprogramming in vitro. Based on the evidence presented above, cancer cells appeared to have induced lipolysis in adipocytes. Thus, to determine the metabolic interaction between adipocytes and lung cancer cells, the metabolic alterations in lung cancer cells in the presence of adipocytes were studied. The results of the Oil Red-O staining pointed to an increased accumulation of lipid droplets in A549 cells following co-culture with adipocytes, suggesting that adipocytes promoted lipid accumulation in lung cancer cells (Fig. 4A). Similarly, compared with cancer cells that were cultured alone, the TG content of A549 cells increased by approximately 1.6 -fold in cells previously co-cultured with adipocytes for 2 days (Fig. 4B; $\mathrm{P}<0.05$ ). Furthermore, a significant increase in glucose uptake and lactate production was observed in A549 cells grown in the presence of Ad-CCM for $24 \mathrm{~h}$, compared with cells cultured in control medium. By contrast, Ad-CM had no effects on the metabolic activity of A549 cells, compared with the control medium cells (Fig. 4C and D). These findings suggested that adipocytes may have altered energy metabolism in lung cancer cells, particularly through glycolysis.

\section{Discussion}

Although a number of potential mechanisms linking adipocytes and cancer have been proposed (26), no conclusive evidence currently exists with regards to the roles of adipocytes and 
A
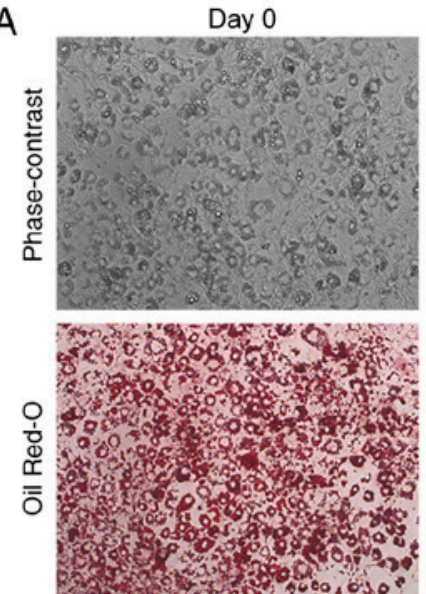

Day 3
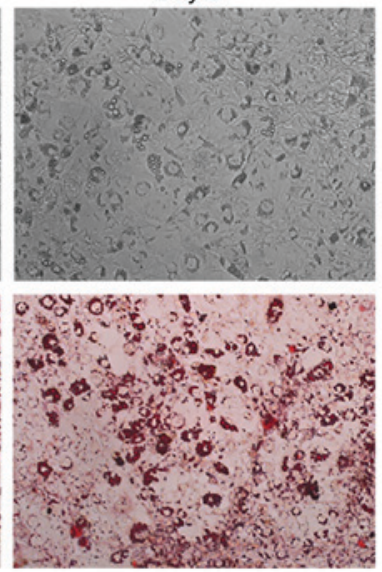

Day 6
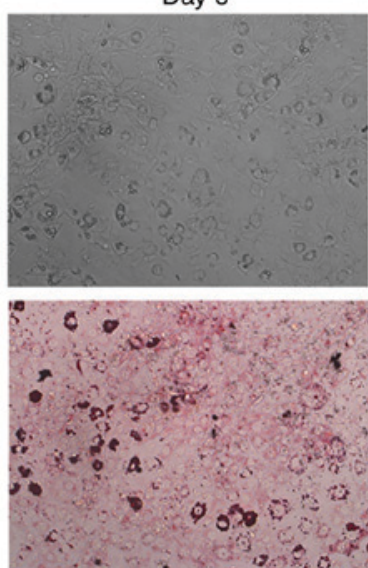

Day 9

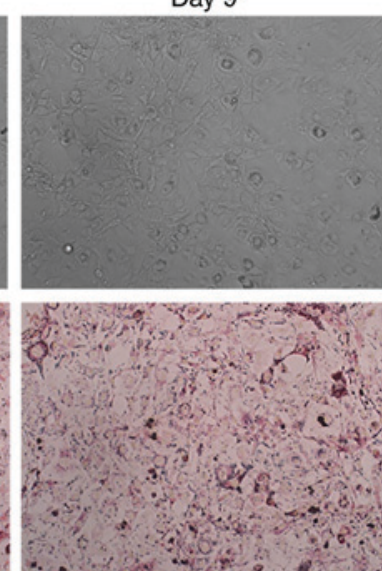

B

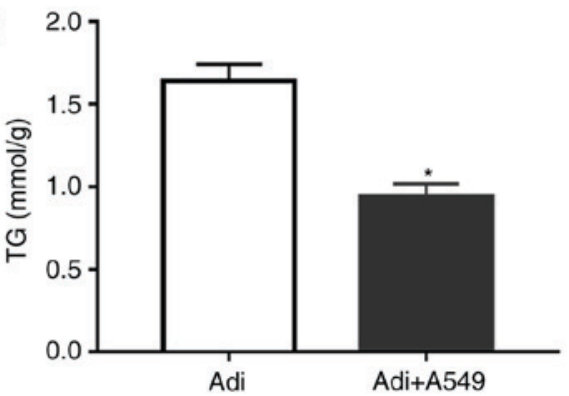

C

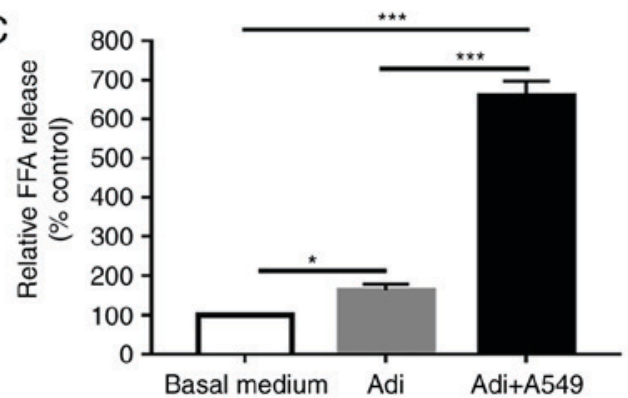

Figure 3. Adipocytes co-cultured with A549 cells exhibit a lipolysis phenotype. (A) Mature adipocytes co-cultured in the presence or absence of A549 cancer cells for the indicated periods were observed by light microscopy and Oil Red-O staining. Magnification, x100. (B) Following indirect co-culture with A549 cells, adipocyte TG content was determined. (C) FFAs were detected in conditioned medium obtained from adipocytes cultured alone or co-cultured with A549 cells. ${ }^{*} \mathrm{P}<0.05$ and ${ }^{* * * *} \mathrm{P}<0.001$. TG, triglyceride; FFAs, free fatty acids; Adi, adipocytes.

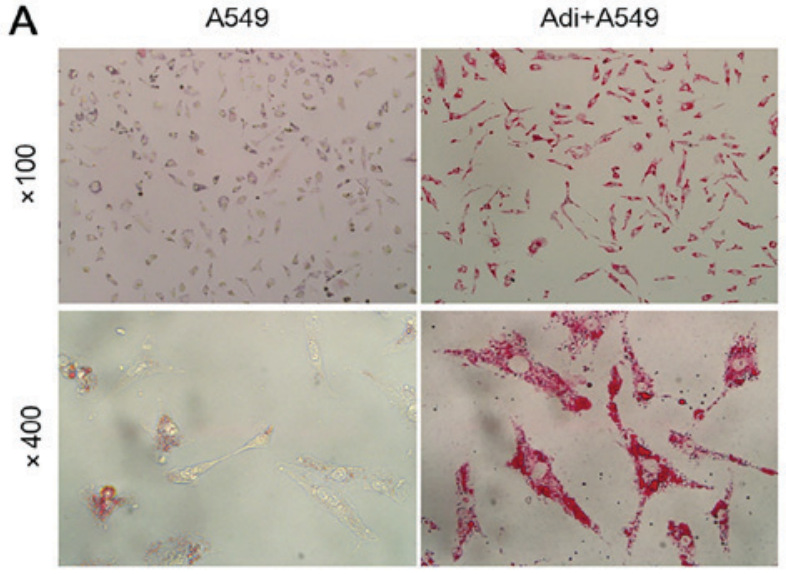

C

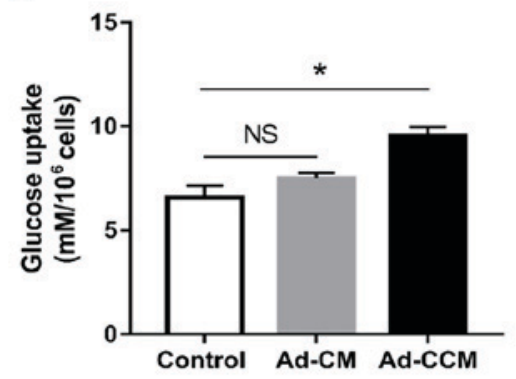

B

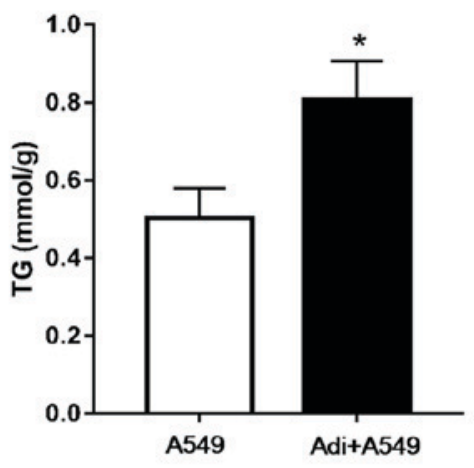

D

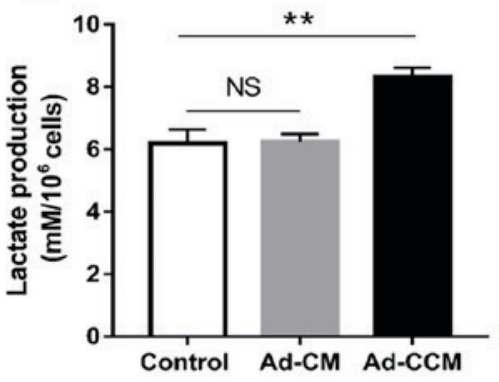

Figure 4. Metabolic remodeling of A549 cells was observed in the presence of adipocytes. (A) A549 cells were indirectly co-cultured in the presence or absence of adipocytes for the indicated periods and observed using Oil Red-O staining. Magnification is indicated on the left. (B) Following indirect co-culture with adipocytes, the TG content in A549 cells was determined. (C) Following treatment with normal medium, Ad-CM or Ad-CCM, glucose uptake and (D) lactate production in A549 cells was detected. ${ }^{*} \mathrm{P}<0.05$ and ${ }^{* *} \mathrm{P}<0.01$. Ad-CM, adipocyte conditioned medium; Ad-CCM, co-cultured adipocyte conditioned medium; NS, not significant; TG, triglyceride; Adi, adipocytes. 
associated metabolic substrates in lung cancer progression. In the present study, the effects of adipocytes on lung adenocarcinoma A549 cells were evaluated using a combination of CM and co-culture approaches. It was demonstrated that following adipocyte interaction, A549 cell proliferation, migration, invasion and intermediary metabolism was significantly altered.

Previous studies have demonstrated that cancer cell proliferation, migration and invasion are altered when they are indirectly co-cultured with adipocytes or exposed to adipocyte CM (6-8,11). Similar effects are observed in xenograft models (27) and 3D cultures (28). However, to the best of our knowledge, no studies have investigated the potential role of adipocytes in lung cancer progression. In the present study, differentiated 3T3-L1 pre-adipocytes were utilized to investigate the crosstalk between adipocytes and A549 lung adenocarcinoma cells. The results demonstrated that the biological behavior of A549 cells cultured in Ad-CM was not notably altered. By contrast, A549 cell metastasis and growth was significantly promoted by Ad-CCM. These findings suggested that crosstalk between adipocytes and cancer cells enhanced the progression of lung cancer. The 3T3-L1 pre-adipocytes used in the present investigation were derived from mouse embryos, not from adult fat tissue. This is due to the limitations of the experimental conditions; at present, it is technically difficult to obtain bone marrow adipocytes and other associated specimens from humans for experimentation. In addition, the functions of adipocytes differ depending on the type of tissue (29). Thus, future research utilizing human omental, subcutaneous and bone marrow adipocytes is required to confirm whether crosstalk exists between adipocytes and lung cancer cells.

Cancer cell proliferation and metastasis are energy-intensive processes and adipocytes store energy in the form of TGs (25). Thus, it was hypothesized that adipocytes provided energy-dense lipids or metabolic substrates for lung adenocarcinoma cells, thereby promoting lung cancer progression. Previous research has demonstrated that when cancer cells invade surrounding adipose tissue, adipocytes exhibit morphological and functional modifications due to enhanced lipolytic activity (5). These modifications include size reduction, increased secretion of certain inflammatory and growth factors, including tumor necrosis factor $\alpha$, interleukin (IL)-6, IL-8 and insulin-like growth factor-1, and decreases in adipocyte-differentiation markers in various types of tumors growing in adipose tissue-dominated microenvironments, including melanoma, gastric, breast, colon, prostate and ovarian cancer (5). In the present study, it was clearly demonstrated that adipocytes co-cultured with A549 cells exhibited a dramatic reduction in the number of lipid droplets and a significant increase in the release of free fatty acids, compared with adipocytes cultured alone. In addition, cytoplasmic lipid droplets accumulated in cancer cells. The latter was consistent with cancer cells acting as metabolic parasites, as demonstrated in a previous study (30). Further research is required to determine whether lipids detected in cancer cells following co-culture are derived from adipocytes or de novo lipogenesis.

Increased glucose uptake and lactate production are characteristics of the Warburg effect, which is the fastest way for cancer cells to produce adenosine 5'-triphosphate via glycolysis (31), allowing them to efficiently gain metabolic autonomy in the tumor microenvironment (31). The resulting production of lactate by highly glycolytic cells is known to enhance the growth of invasive cancer cells (32). In addition, lactate may serve as an alternative carbon source for the surrounding oxygenated cells (32). Previous research has demonstrated that bone marrow adipocytes promote the expression of glycolytic enzymes, increase lactate production and decrease mitochondrial oxidative phosphorylation (15). The present study additionally demonstrated that adipocytes were capable of altering the Warburg phenotype in A549 cells through paracrine signaling: A549 cells cultured in Ad-CCM consumed more glucose and produced more lactate, compared with A549 cells cultured in Ad-CM or normal medium. A previous report indicated that the interaction between adipocytes and cancer cells ultimately affects cancer cell metabolism, allowing for adaptive survival in the metastatic niche (33).

Previous literature and the findings of the present study highlight the potential for characterization of adipocyte-cancer cell interactions, to provide a better understanding of lung cancer progression, and to identify avenues for the development of novel therapeutic strategies.

\section{Acknowledgements}

The authors thank Professor Shan Gao (Department of Pharmacology, Basic Medical College, Anhui Medical University) for his technical assistance.

\section{Funding}

The present study was supported by the National Natural Science Foundation of China (grant no. 81402427) and the Provincial Natural Science Foundation of Anhui (grant no. 1408085QH165).

\section{Availability of data and materials}

The datasets used and/or analyzed during the current study are available from the corresponding author on reasonable request.

\section{Authors' contributions}

FFL conceived, designed and supervised the study. HZ made the experiment, analyzed and interpreted the data, and was a principal contributor in writing the manuscript. JJL and YNC performed the partial experiments. XD and CG performed the data collection and participated in the writing of the manuscript. All authors read and approved the final manuscript.

\section{Ethics approval and consent to participate}

Not applicable.

\section{Consent for publication}

Not applicable.

\section{Competing interests}

The authors declare that they have no competing interests. 


\section{References}

1. Miller KD, Siegel RL, Lin CC, Mariotto AB, Kramer JL, Rowland JH, Stein KD, Alteri R and Jemal A: Cancer treatment and survivorship statistics, 2016. CA Cancer J Clin 66: 271-289, 2016.

2. Chalela R, Curull V, Enriquez C, Pijuan L, Bellosillo B and Gea J: Lung adenocarcinoma: From molecular basis to genome-guided therapy and immunotherapy. J Thorac Dis 9: 2142-2158, 2017.

3. Divella R, De Luca R, Abbate I, Naglieri E and Daniele A: Obesity and cancer: The role of adipose tissue and adipo-cytokines-induced chronic inflammation. J Cancer 7: 2346-2359, 2016.

4. Gucalp A, Iyengar NM, Hudis CA and Dannenberg AJ: Targeting obesity-related adipose tissue dysfunction to prevent cancer development and progression. Semin Oncol 43: 154-160, 2016.

5. Nieman KM, Romero IL, Van Houten B and Lengyel E: Adipose tissue and adipocytes support tumorigenesis and metastasis Biochim Biophys Acta 1831: 1533-1541, 2013.

6. Laurent V, Guérard A, Mazerolles C, Le Gonidec S, Toulet A, Nieto L, Zaidi F, Majed B, Garandeau D, Socrier Y, et al: Periprostatic adipocytes act as a driving force for prostate cancer progression in obesity. Nat Commun 7: 10230, 2016.

7. Nieman KM, Kenny HA, Penicka CV, Ladanyi A, Buell-Gutbrod R, Zillhardt MR, Romero IL, Carey MS Mills GB, Hotamisligil GS, et al: Adipocytes promote ovarian cancer metastasis and provide energy for rapid tumor growth. Nat Med 17: 1498-1503, 2011.

8. Lazar I, Clement E, Dauvillier S, Milhas D, Ducoux-Petit M, LeGonidec S, Moro C, Soldan V, Dalle S, Balor S, et al: Adipocyte exosomes promote melanoma aggressiveness through fatty acid oxidation: A novel mechanism linking obesity and cancer. Cancer Res 76: 4051-4057, 2016.

9. Balaban S, Shearer RF, Lee LS, van Geldermalsen M, Schreuder M, Shtein HC, Cairns R, Thomas KC, Fazakerley DJ, Grewal T, et al: Adipocyte lipolysis links obesity to breast cancer growth: adipocyte-derived fatty acids drive breast cancer cel proliferation and migration. Cancer Metab 5: 1, 2017.

10. Rajala MW and Scherer PE: Minireview: The adipocyte-at the crossroads of energy homeostasis, inflammation, and atherosclerosis. Endocrinology 144: 3765-3773, 2003

11. Dirat B, Bochet L, Dabek M, Daviaud D, Dauvillier S Majed B, Wang YY, Meulle A, Salles B, Le Gonidec S, et al: Cancer-associated adipocytes exhibit an activated phenotype and contribute to breast cancer invasion. Cancer Res 71: 2455-2465, 2011.

12. Warburg O: On respiratory impairment in cancer cells. Science 124: 269-270, 1956.

13. Manzi L, Costantini L, Molinari R and Merendino N: Effect of dietary $\omega-3$ polyunsaturated fatty acid DHA on glycolytic enzymes and warburg phenotypes in cancer. Biomed Res Int 2015: 137097, 2015.

14. Choi SY, Collins CC, Gout PW and Wang Y: Cancer-generated lactic acid: A regulatory, immunosuppressive metabolite? J Pathol 230: 350-355, 2013.

15. Diedrich JD, Rajagurubandara E, Herroon MK, Mahapatra G, Hüttemann M and Podgorski I: Bone marrow adipocytes promote the Warburg phenotype in metastatic prostate tumors via HIF-1 $\alpha$ activation. Oncotarget 7: 64854-64877, 2016.

16. Baenke F, Peck B, Miess H and Schulze A: Hooked on fat: The role of lipid synthesis in cancer metabolism and tumour development. Dis Model Mech 6: 1353-1363, 2013.
17. Yan L and DeMars LC: Effects of dietary fat on spontaneous metastasis of Lewis lung carcinoma in mice. Clin Exp Metastasis 27: 581-590, 2010

18. Cao N, Ma X, Guo Z, Zheng Y, Geng S, Meng M, Du Z, Lin H, Duan Y and Du G: Oral kanglaite injection (KLTI) attenuates the lung cancer-promoting effect of high-fat diet (HFD)-induced obesity. Oncotarget 7: 61093-61106, 2016.

19. Kuchuk M, Addison CL, Clemons M, Kuchuk I and Wheatley-Price P: Incidence and consequences of bone metastases in lung cancer patients. J Bone Oncol 2: 22-29, 2013.

20. Jones JG: Hepatic glucose and lipid metabolism. Diabetologia 59: 1098-1103, 2016

21. Johnson AC, McNabb AR and Rossiter RJ: Lipids of normal brain. Biochem J 43: 573-577, 1948.

22. Basinska J, Sastry PS and Stancer HC: Lipid composition of human, bovine and sheep pineal glands. J Neurochem 16: 707-714, 1969.

23. Paget S: The distribution of secondary growths in cancer of the breast. 1889. Cancer Metastasis Rev 8: 98-101, 1989.

24. Coelho P, Almeida J, Prudencio C, Fernandes R and Soares R: Effect of adipocyte secretome in melanoma progression and vasculogenic mimicry. J Cell Biochem 117: 1697-1706, 2016.

25. Shafat MS, Oellerich T, Mohr S, Robinson SD, Edwards DR, Marlein CR, Piddock RE, Fenech M, Zaitseva L, Abdul-Aziz A, et al: Leukemic blasts program bone marrow adipocytes to generate a protumoral microenvironment. Blood 129: 1320-1332, 2017.

26. Duong MN, Geneste A, Fallone F, Li X, Dumontet C and Muller C: The fat and the bad: Mature adipocytes, key actors in tumor progression and resistance. Oncotarget 8: 57622-57641, 2017.

27. Wang C, Gao C, Meng K, Qiao H and Wang Y: Human adipocytes stimulate invasion of breast cancer MCF-7 cells by secreting IGFBP-2. PLoS One 10: e0119348, 2015.

28. Lapeire L, Hendrix A, Lambein K, Van Bockstal M, Braems G, Van Den Broecke R, Limame R, Mestdagh P, Vandesompele J, Vanhove $\mathrm{C}$, et al: Cancer-associated adipose tissue promotes breast cancer progression by paracrine oncostatin $\mathrm{M}$ and Jak/STAT3 signaling. Cancer Res 74: 6806-6819, 2014.

29. Ibrahim MM: Subcutaneous and visceral adipose tissue: Structural and functional differences. Obes Rev 11: 11-18, 2010.

30. Martinez-Outschoorn UE, Pestell RG, Howell A, Tykocinski ML, Nagajyothi F, Machado FS, Tanowitz HB, Sotgia F and Lisanti MP: Energy transfer in 'parasitic' cancer metabolism: Mitochondria are the powerhouse and Achilles' heel of tumor cells. Cell Cycle 10: 4208-4216, 2011.

31. Chiche J, Brahimi-Horn MC and Pouyssegur J: Tumour hypoxia induces a metabolic shift causing acidosis: A common feature in cancer. J Cell Mol Med 14: 771-794, 2010.

32. Sonveaux P, Végran F, Schroeder T, Wergin MC, Verrax J, Rabbani ZN, De Saedeleer CJ, Kennedy KM, Diepart C, Jordan BF, et al: Targeting lactate-fueled respiration selectively kills hypoxic tumor cells in mice. J Clin Invest 118: 3930-3942, 2008.

33. Martinez-Outschoorn UE, Sotgia F and Lisanti MP: Power surge: Supporting cells 'fuel' cancer cell mitochondria. Cell Metab 15: $4-5,2012$.

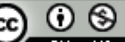

This work is licensed under a Creative Commons Attribution 4.0 International (CC BY-NC 4.0) License 